\title{
Modifizierte Abdeckung für Eingriffe in Steinschnittlage
}

\author{
Wie Sie ein Standard-OP-Abdeckungsset für Eingriffe in Steinschnittlage nutzen können, \\ zeigen wir Ihnen in folgendem Tipp.
}

Ein Standard-OP-Abdeckungsset enthält in der Regel 1 großes Tuch für die Kopfseite („Anästhesietuch“), 1 kleineres Tuch für die Fußseite, 2 Seitentücher sowie Tischbezüge und Klebetape. Für Eingriffe in Steinschnittlage werden zusätzlich 2 Beinlinge benötigt. In unserer Hand hat sich ein kleiner Trick bewährt, diese Elemente der Abdeckung so zu nutzen, dass eine wasserdichte Schürze zum Operateur entsteht, die Beinlinge gerafft und die Übersicht verbessert wird, besonders, wenn 2 Operateure am Tisch sind.

Ein Seitentuch wird als wasserdichtes „Unterlegetuch“ unter den Patienten geschoben ( $\triangle$ Abb. 1 ) und die Beinlinge angebracht ( $\triangle$ Abb. 2 und $\odot$ Abb. 3). Nach oben wird nun das kleinere, eigentlich für die Abdeckung nach unten vorgesehene
Tuch aufgeklebt ( $\triangle$ Abb.4). Nach unten wird - entgegen der ursprünglichen Bestimmung - das große „Anästhesietuch“ auf das Perineum geklebt ( $\bullet$ Abb.5). Es bleibt gefaltet, wird um die Beine herumgeführt und an seinen beiden Enden in der Mitte über dem Bauch des Patienten mit einer Klemme vereinigt ( $\triangle$ Abb. 6 und - Abb. 7). Aus dem noch gefalteten Teil des nun unten klebenden „Anästhesietuchs“ kann durch Herausziehen und Befestigen am Kittel eine wasserdichte Schürze oder eine Ablage für Instrumente, Tupfer o.ä. entstehen. Die Beinlinge können mit jeweils einem halben Klebetape am Oberschenkel keimdicht versiegelt werden (o Abb. 8).

\section{Karsten Eisenblätter,}

PD Dr. Andreas Wiedemann, Witten
Korrespondenz:

PD Dr. Andreas Wiedemann

Urologische Klinik

Evangelisches Krankenhaus

im Diakoniewerk Ruhr gGmbH

Lehrstuhl für Geriatrie

der Universität Witten/Herdecke

Pferdebachstr. 27

58455 Witten

Tel.: +49/2302/175-2521

awiedemann@diakonie-ruhr.de

Interessenkonflikte: PD Dr. Wiedemann: Beratungstätigkeit: Dr. Pfleger, Pfizer; Vortragstätigkeit: Allergan, AMS Deutschland, Astellas, Berlin-Chemie, Jansen, Lilly Deutschland, Dr. Pfleger, Pfizer, PohlBoskamp; Studienfinanzierung: AMS Deutschland

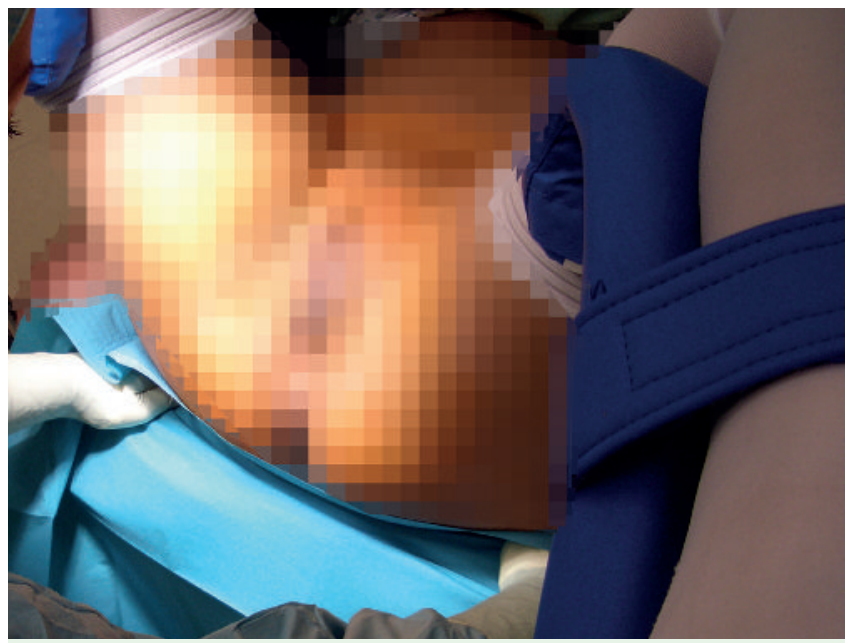

Abb. 1 Das wasserdichte Unterlegtuch wird positioniert.

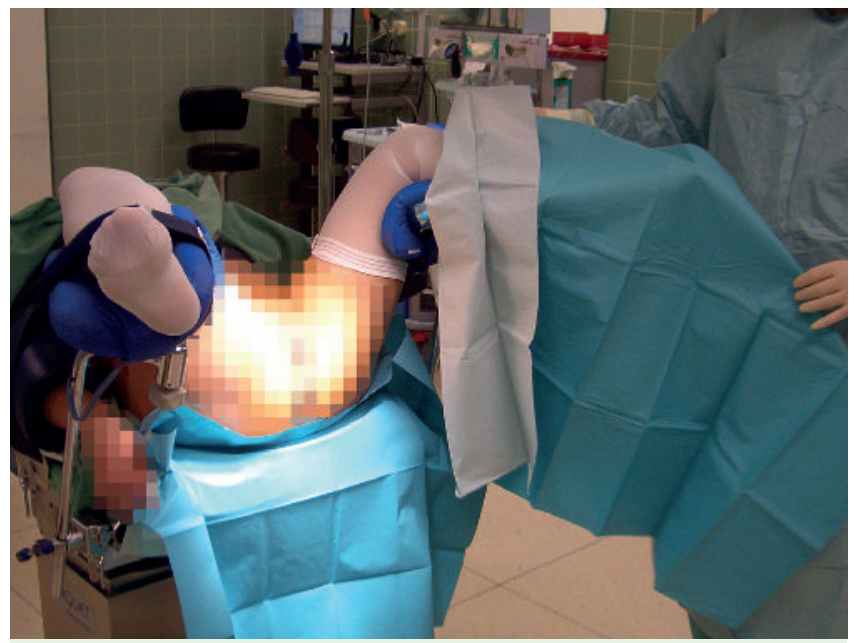

Abb. 2 Anbringen der Beinlinge. 


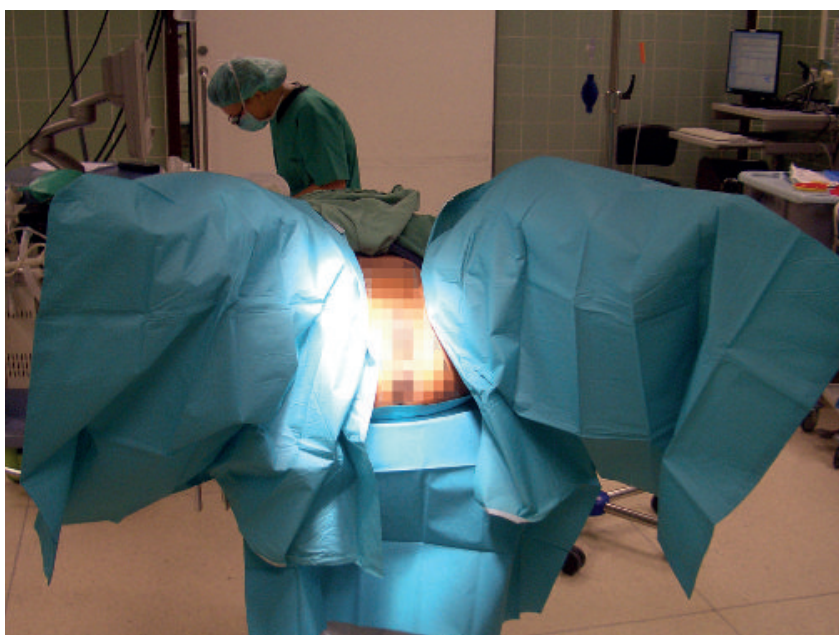

Abb. 3 Beinlinge und Unterlegtuch in Position.

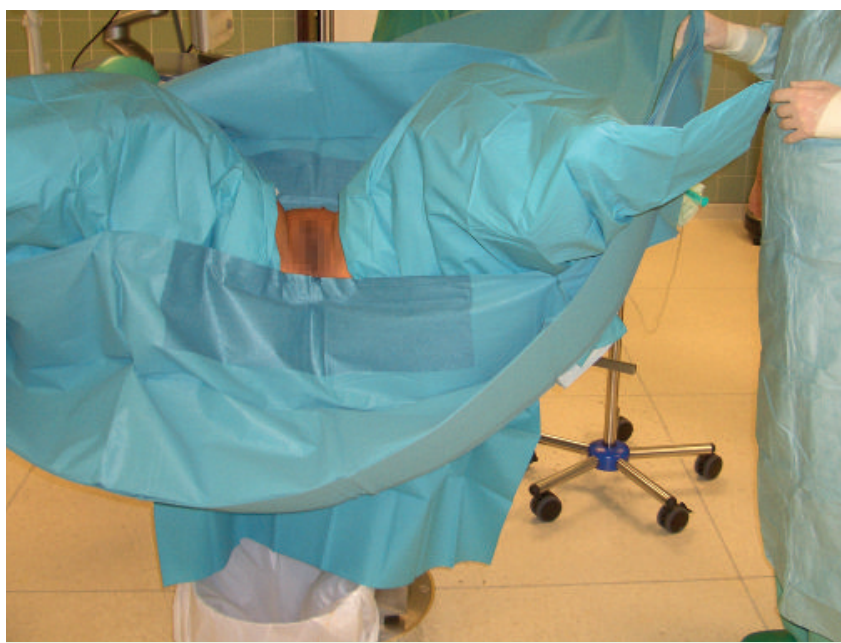

Abb. 5 Das größere „Anästhesietuch“ wird entgegen seiner eigentlichen Bestimmung nach unten geklebt und um die Beine herumgeführt ...

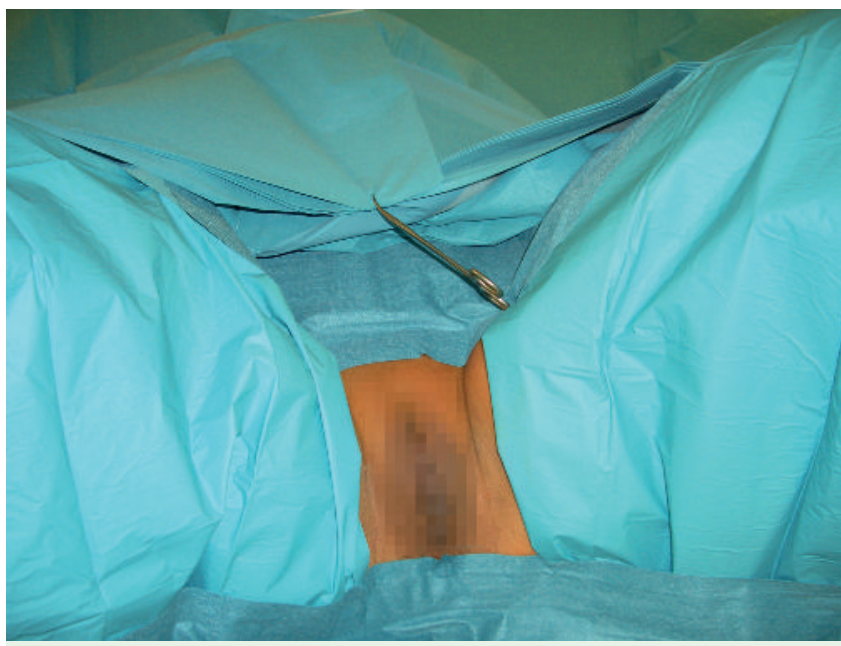

Abb. 7 Fertige modifizierte Abdeckung.

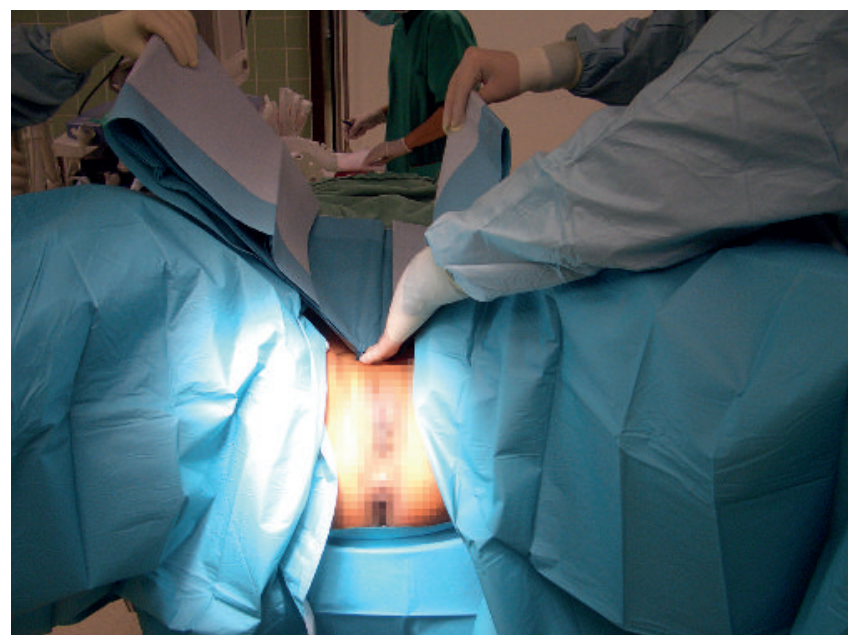

Abb. 4 Das kleinere Tuch zur Abdeckung des unteren Teils des Situs wird als „Anästhesietuch“ nach oben geklebt.

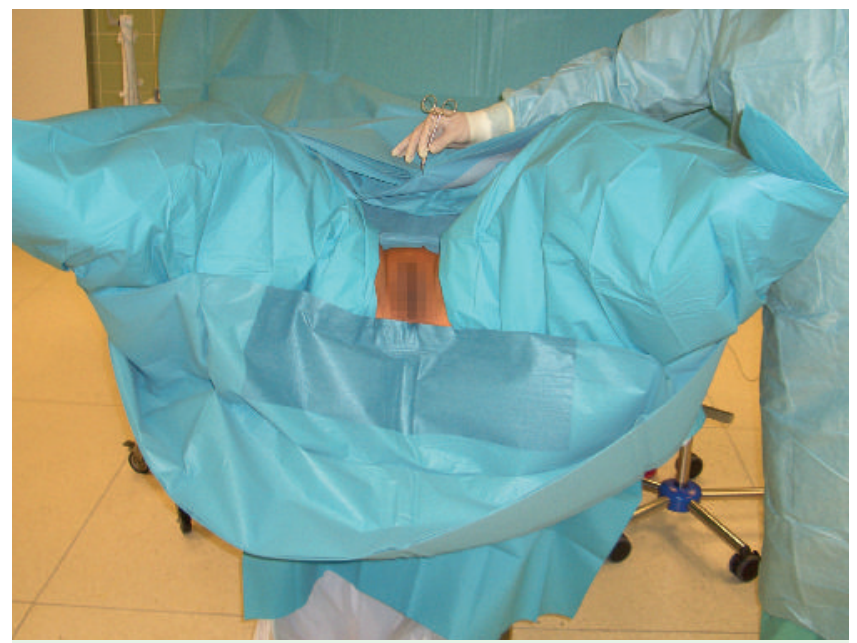

Abb. 6 ...und seine beiden Enden in der Mitte mit einer Klemme vereinigt.

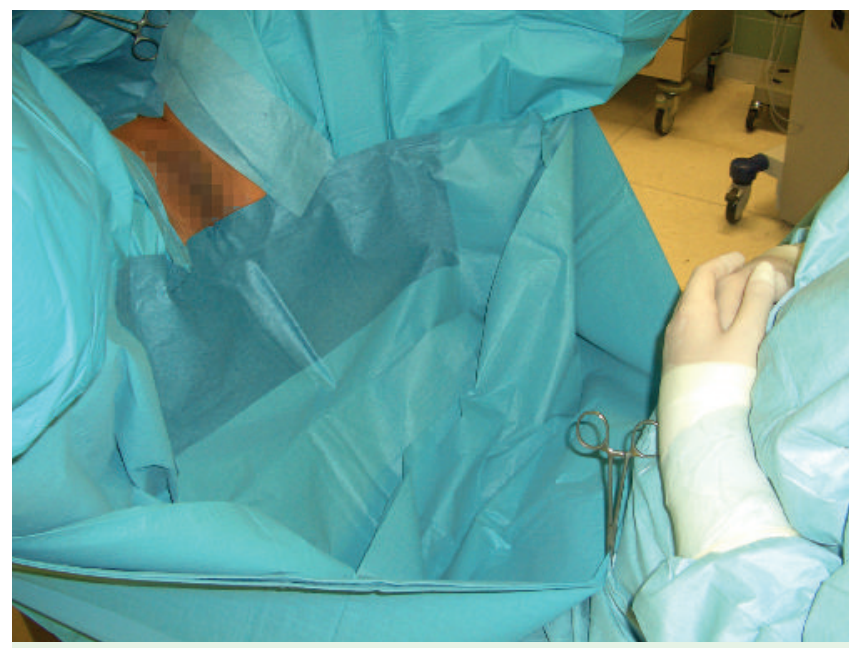

Abb. 8 Durch Herausziehen der Falten und Befestigen am Kittel des Operateurs ergibt sich eine Arbeitsplattform („Schürze“). Am linken Oberschenkel ist der keimdichte Abschluss und die Fixierung des Beinlings mit einem halben Tape zu sehen. 